\title{
Post-SL9 sulfur photochemistry on Jupiter
}

\author{
Julianne I. Moses \\ Lunar and Planetary Institute, Houston, Texas \\ Mark Allen \\ Jet Propulsion Laboratory, Pasadena, California \\ G. Randall Gladstone \\ Southwest Research Institute, San Antonio, Texas
}

\begin{abstract}
We have modeled the photochemical evolution of the sulfur-containing species that were observed in Jupiter's stratosphere after the SL9 impacts. We find that most of the sulfur is converted to $S_{8}$ in the first few days. Other important sulfur reservoirs are CS, whose abundance increases markedly with time, and possibly $\mathrm{H}_{2} \mathrm{CS}$, HNCS, and NS, whose abundances depend on kinetic reaction rates that are unknown at the present. We discuss the temporal variation of the major sulfur compounds, make abundance and compositional predictions useful for comparisons with observations, and discuss the possible condensation of sulfur-containing species.
\end{abstract}

\section{Introduction}

Observational reports indicate that many new molecular species were deposited in Jupiter's stratosphere following the Comet P/Shoemaker-Levy 9 (SL9) impacts. These new species derive from highly processed cometary material and tropospheric Jovian air that were modified during the impact explosion, fireball, and re-entry shock ("splashdown") of the plume back into the upper atmosphere. To examine the photochemical evolution of the species that were observed or predicted to have formed following the plume splashdown ( $\sim 10-15$ minutes after the actual impact), we use a onedimensional (1-D) chemical kinetics and diffusion model similar to the one described in Yung et al. [1984]. Our results regarding the sulfur-containing species are presented at this time - results regarding nitrogen and oxygen species are presented in a companion paper [Moses et al., 1995].

The Hubble Space Telescope (HST) Faint Object Spectrograph (FOS) obtained ultraviolet spectra within the central dark core of the G (and L) site over a period of several days and weeks following the impacts [Noll et al., 1995; Yelle and McGrath, 1995]. These highquality spectra indicate the presence of several sulfur compounds at the site: $\mathrm{S}_{2}, \mathrm{CS}_{2}, \mathrm{CS}$, and $\mathrm{H}_{2} \mathrm{~S}$ are all

Copyright 1995 by the American Geophysical Union.

Paper number 95GL01200 0094-8534/95/95GL-01200\$03.00 identified; notably absent are signatures for $\mathrm{SO}_{2}$ and SO. Although $\mathrm{SO}_{2}$ has been detected in mid-infrared spectra [A. Sprague, personal communication, 1994], its inferred abundance is several orders of magnitude below that of the other observed sulfur species. These observations suggest that oxygen compounds may not have played a large role in the impact chemistry [cf. Zahnle et al. 1995]. On the other hand, several Earthbased observations imply that oxygen species such as $\mathrm{H}_{2} \mathrm{O}$ and $\mathrm{CO}$ were abundant at the same $\mathrm{G}$ impact site [Lellouch et al., 1995; Bjoraker et al., 1994; Meadows et al., 1994]. Because the reason for the discrepancy between the HST and other observations is not known, we concentrate on the dark core regions observed by the FOS and assume that the oxygen compounds remain within the HST upper limits.

The complex nature of the impacts makes it impossible for 1-D photochemical models to reproduce all the phenomena that affect the impact debris; however, such models are useful for several reasons. First of all, the models can be used to predict the temporal variation of the initially observed species; the degree to which the predictions match the observations tells us about the relative effectiveness of dynamical vs. photochemical processes in the post-SL9 Jovian stratosphere and can greatly enhance our understanding of complex photochemical mechanisms that are unstudied in laboratories on Earth. Secondly, the models can be used to direct the search for undetected molecular species and to identify the short- and long-term reservoirs of various elements. Finally, such models can aid in the analysis of the haze observations by predicting the composition, location, formation rate, and lifetime of possible condensates in Jupiter's stratosphere.

\section{Photochemical Model}

The hydrocarbon reaction list, absorption cross sections, quantum yields, diffusion coefficients, boundary conditions, and background atmospheric structure of Gladstone et al. [1995] form the basis for our photochemical model, but we update the hydrocarbon photochemistry and include oxygen, nitrogen, and sulfur reactions. Our photochemical model contains 144 species and over 900 reactions. We have tried to identify and include the important reaction schemes that are most 
likely to affect the abundances of the "parent" molecules observed after the SL9 impacts. However, the rates for several important reactions are not available in the literature, and we often had to make estimates. Therefore, this work represents a preliminary, qualitative attempt to follow the evolution of the important chemical species; the results should not be regarded as definitive. Our reaction list is available upon request.

Since some of the observed species form most easily in low pressure re-entry shocks [Zahnle et al., 1995], we assumed that most of the new material was deposited above $1 \mathrm{mbar}$ in the stratosphere. Based on the results presented by Noll et al. [1994], we start with initial column abundances of reduced sulfur species as shown in Table 1. The initial abundances of the nitrogen- and oxygen-containing compounds are described in Moses et al. [1995]. Due to a lack of coherent information at this time, we do not enhance the hydrocarbon abundances after the plume splashdown, but begin with the background hydrocarbon abundances derived by Gladstone et al. [1995] for the same latitude region. The new postimpact photochemistry does not measurably affect the profiles of the major hydrocarbon compounds.

\section{Results}

The abundances of our initial sulfur species change with time as photochemical reactions are initiated. Table 1 illustrates the temporal variation of several of the more interesting species. Due to the large uncertainties in initial compositions at the impact sites, one should not put great reliance on the actual numbers in this table; two significant digits are included solely as an aid in comparing relative changes.

\section{Sulfur Photochemistry}

The most abundant sulfur compound observed immediately following the impacts is diatomic sulfur $\left(\mathrm{S}_{2}\right)$. $\mathrm{S}_{2}$ is quickly lost by photolysis in the upper atmosphere and by the formation of larger sulfur molecules in the lower stratosphere. In fact, the production of molecular sulfur $\left(\mathrm{S}_{8}\right)$ by various three-body reactions is very rapid. We adopt the following scheme for the production of various sulfur molecules:

$$
\begin{array}{rllr}
\mathrm{S}_{\mathrm{x}} & \stackrel{h \nu}{\longrightarrow} & \mathrm{S}_{\mathrm{x}-1}+\mathrm{S} & \text { for } \mathrm{x}=2,3,4 \\
2 \mathrm{~S} & \stackrel{\mathrm{M}}{\longrightarrow} \mathrm{S}_{2} & \\
\mathrm{~S}+\mathrm{S}_{2} & \stackrel{\mathrm{M}}{\longrightarrow} \mathrm{S}_{3} & \\
2 \mathrm{~S}_{3} & \stackrel{\mathrm{M}}{\longrightarrow} & \mathrm{S}_{6} & \\
\mathrm{~S}_{2}+\mathrm{S}_{\mathrm{x}} & \stackrel{\mathrm{M}}{\mathrm{S}_{\mathrm{x}+2}} & \text { for } \mathrm{x}=2,4,6 \\
\mathrm{~S}+\mathrm{S}_{\mathrm{x}} & \longrightarrow & \mathrm{S}_{2}+\mathrm{S}_{\mathrm{x}-1} & \text { for } \mathrm{x}=3,4,5,6 \\
2 \mathrm{~S}_{4} & \stackrel{\mathrm{M}}{\longrightarrow} & \mathrm{S}_{8} &
\end{array}
$$

where $\mathrm{M}$ represents any third molecule or atom. As Table 1 indicates, over $70 \%$ of the initial column budget of $\mathrm{S}_{2}$ molecules has been converted to $\mathrm{S}_{8}$ in the first hour after we begin our calculations, and another $\sim 10 \%$ is converted to other sulfur molecules (e.g., $S_{3}, S_{4}$ ). $S_{8}$, which has a puckered ring structure, will condense almost as soon as it is formed.
On the other hand, $S_{8}$ formation is only rampant in the middle and lower stratosphere at pressures greater than $\sim 0.1$ mbar. At higher altitudes, $S_{2}$ is effectively photolyzed before larger sulfur molecules can form. The $\mathrm{S}_{2}(\mathrm{~B}-\mathrm{X})$ band system lies at $240-360 \mathrm{~nm}$ and is clearly observed by the HST FOS [Noll et al., 1995]. At wavelengths shorter than $\sim 278 \mathrm{~nm}, \mathrm{~S}_{2}$ dissociates into two ground-state $\left({ }^{3} \mathrm{P}\right)$ sulfur atoms. The lifetime against photolysis for the $S_{2}$ molecule at $10^{-5}$ mbar and $44^{\circ} \mathrm{S}$ latitude on Jupiter in our diurnally averaged model is 5.3 hours. At high altitudes, photolysis seems to provide an effective permanent loss mechanism for the $\mathrm{S}_{2}$; however, in the middle stratosphere near $0.01-0.1 \mathrm{mbar}$, the three-body reaction $2 \mathrm{~S}+\mathrm{M} \rightarrow \mathrm{S}_{2}+\mathrm{M}$ balances the photolysis, and $S_{2}$ is efficiently recycled. Thus, we find that some $S_{2}$ remains several months after the impacts (see Fig. 1 and Table 1). Because this result depends on the rates of several reactions that have not been measured in the laboratory, this conclusion should be regarded as speculative. The HST spectra show no evidence for $S_{2}$ at the $G$ site 22 days after the impact, but no upper limits are given in Noll et al. [1995], and it is unclear whether models such as are shown in Fig. 1, in which the $S_{2}$ is confined to a narrow altitude region, are consistent with these observations.

Carbon disulfide $\left(\mathrm{CS}_{2}\right)$, which is also observed unambiguously in the IIS' $\Gamma$ FOS spectra, has strong absorption bands in the $185-230 \mathrm{~nm}$ region. At these wavelengths, $\mathrm{CS}_{2}$ dissociates into $\mathrm{CS}+\mathrm{S}$, where $\sim 80 \%$ of the sulfur atoms emerge in the ${ }^{1} \mathrm{D}$ excited state and the rest in the ${ }^{3} \mathrm{P}$ ground state. The $S\left({ }^{1} \mathrm{D}\right)$ reacts efficiently with $\mathrm{H}_{2}$ to form $\mathrm{SH}+\mathrm{H}$. $\mathrm{CS}_{2}$, like $\mathrm{S}_{2}$, is not lost from the middle stratosphere as fast as its photolysis lifetime ( 8.2 hours at $10^{-5}$ mbar) would indicate. Instead, $\mathrm{CS}_{2}$ is partly recycled and is also formed during the photolysis of $S_{2}$ in reaction schemes that involve $\mathrm{S}$ atoms and hydrocarbon radicals and have $\mathrm{H}_{2} \mathrm{CS}$ and HCS as intermediates.

Thioformaldehyde $\left(\mathrm{H}_{2} \mathrm{CS}\right)$ forms in potentially observable quantities if we assume (as we did) that the

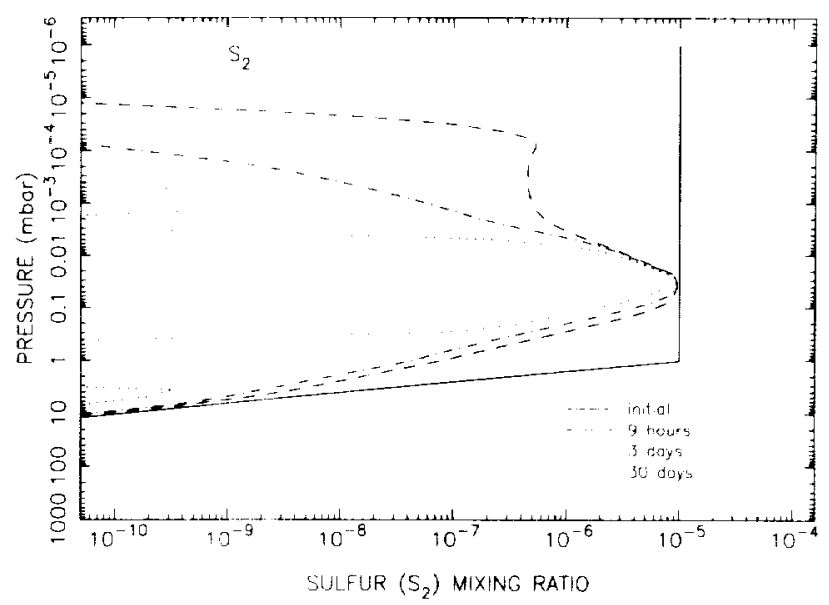

Figure 1. The evolution of $S_{2}$. The solid line shows our assumed initial abundance, the dashed line shows the mixing ratio after 9 hours, the dot-dashed line after 3 days, and the dotted line after 30 days. 
unmeasured reaction $\mathrm{S}+\mathrm{CH}_{3} \rightarrow \mathrm{H}_{2} \mathrm{CS}+\mathrm{H}$ is fast. It is possible, however, that $\mathrm{S}+\mathrm{CH}_{3}$ will act to preferentially form $\mathrm{CH}_{3} \mathrm{~S}$ via three-body reactions and that $\mathrm{H}_{2} \mathrm{CS}$ will not be a major product. If this alternative is true, then $\mathrm{H}_{2} \mathrm{CS}$ will cease to be a major component in our model while methyl mercaptan $\left(\mathrm{CH}_{3} \mathrm{SH}\right)$, dimethyl sulfide $\left(\mathrm{CH}_{3} \mathrm{SCH}_{3}\right)$, dimethyl disulfide $\left(\mathrm{CH}_{3} \mathrm{~S}\right)_{2}$, and organic sulfur ring species (e.g., $\mathrm{C}_{2} \mathrm{H}_{4} \mathrm{~S}, \mathrm{C}_{3} \mathrm{H}_{6} \mathrm{~S}$ ) will become more important. CS production would also decrease in this case.

Carbon sulfide (CS) is produced by $\mathrm{CS}_{2}$ photolysis and, more importantly in our model, by several different reaction schemes whose ultimate source is $S_{2}$ photolysis. Although CS is considered a radical species, very few reactions seem to be effective at permanently removing it from Jupiter's stratosphere. One exception is a reaction that we have invented: $\mathrm{NH}_{2}+\mathrm{CS} \rightarrow \mathrm{HNCS}+\mathrm{H}$. We have assumed that this reaction proceeds relatively inefficiently, but the CS and the $\mathrm{NH}_{2}$ radicals (which result from $\mathrm{NH}_{3}$ photolysis) are so abundant that the reaction becomes important. As can be seen from Table 1, the abundance of isothiocyanic acid (HNCS) increases dramatically with time. We have not included any loss mechanisms for HNCS in our model, so if it does not condense in the stratosphere, it may be photolyzed before significant amounts can build up.

Our prediction for the CS column abundance 3 days after the impact is $2 \times 10^{15} \mathrm{~cm}^{-2}$, which is an order of magnitude higher than the abundance derived by Noll et al. [1995] for CS observed in emission by the HST FOS three days after the $G$ impact. However, our results do compare well with the millimeter observations of Lellouch et al. [1995] also taken 3 days after the G impact if we assume that the $\sim 3 \times 10^{11} \mathrm{~g}$ CS seen by these authors is spread over an areal spot $\sim 7500 \mathrm{~km}$ in radius. The column quoted by Noll et al. would require the CS to be spread over a spot $25,000 \mathrm{~km}$ in radius in order to account for the Lellouch et al. observations.

Hydrogen sulfide $\left(\mathrm{H}_{2} \mathrm{~S}\right)$ is inferred, but not unambiguously detected, in the HST FOS absorption spectra [Yelle and McGrath, 1995; Noll et al., 1995]. $\mathrm{H}_{2} \mathrm{~S}$ has a nearly featureless absorption in the $165-250 \mathrm{~nm}$ region, with a broad peak located near $187 \mathrm{~nm}$. Photolysis of $\mathrm{H}_{2} \mathrm{~S}$ proceeds predominantly by $\mathrm{H}_{2} \mathrm{~S}+h \nu \rightarrow \mathrm{H}+\mathrm{SH}$; the $\mathrm{H}_{2} \mathrm{~S}$ lifetime against photolysis is 1.8 days at $10^{-5}$ mbar. The $\mathrm{SH}$ radicals formed during the $\mathrm{H}_{2} \mathrm{~S}$ photolysis react with atomic $\mathrm{H}$ in the upper atmosphere to form $\mathrm{S}+\mathrm{H}_{2}$ and with various sulfur radicals in the lower stratosphere to form $\mathrm{HS}_{2} . \mathrm{H}_{2} \mathrm{~S}$ is not efficiently recycled and is lost rapidly from the stratosphere.

A close examination of Table 1 will reveal that nitrogen-sulfur species are important reservoirs for the sulfur. This result is one of the most speculative of our paper. Sulfur-nitrogen reactions have not been extensively studied in laboratories, but both of the main N-S species in our model, NS radicals and HNCS molecules, have been observed in interstellar space. In our model, HNS radicals are produced through reactions of atomic sulfur with $\mathrm{NH}_{2}$. The HNS radicals react with both $\mathrm{S}$ and $\mathrm{SH}$ to form NS, but the only permanent loss for NS in our model is the fast reaction $\mathrm{N}+\mathrm{NS} \rightarrow \mathrm{N}_{2}+\mathrm{S}$. Since $\mathrm{N}$ atoms are not abundant, this reaction is ineffective. As mentioned previously, HNCS is formed by the reaction of $\mathrm{NH}_{2}$ with $\mathrm{CS}$. If the reactions producing these species are ineffective or if these species are readily lost by mechanisms we have omitted, then our conclusions regarding the major reservoirs of both sulfur and nitrogen may change. The sulfur will still mostly end up in $\mathrm{S}_{8}$, but this process may be accelerated, and ammonia $\left(\mathrm{NH}_{3}\right)$ may be more efficiently recycled and not lost so rapidly from our model [see Moses et al., 1995].

\section{Condensates}

Dark aerosol particles were immediately visible at the impact sites [e.g., West et al., 1994]. These particles probably consist of silicates, metal sulfides or oxides, and/or organic debris (e.g., soot) that formed thermochemically in the impact or re-entry shocks. Does photochemistry contribute to the aerosol properties? The particles themselves are very dark [West et al., 1995], and none of the species in our model form condensates that are as dark. In an interesting and relevant laboratory experiment, Khare et al. [1978] irradiated a mixture of $\mathrm{CH}_{4}, \mathrm{NH}_{3}$, and $\mathrm{H}_{2} \mathrm{~S}$ gases with high doses of $254 \mathrm{~nm}$ ultraviolet radiation. The brown solid that formed during this procedure was composed of $84 \% \mathrm{~S}_{8}$ and $16 \%$ more complex organic matter. This experiment, and others like it, reveal that solids that contain a large percentage of $\mathrm{S}_{8}$ do not necessarily have to be bright at visible wavelengths.

Table 1. Time Variation of Column Abundances $\left(\mathrm{cm}^{-2}\right)$

\begin{tabular}{|c|c|c|c|c|c|c|}
\hline Species & Initial & $1 \mathrm{hr}$ & 3 days & 9 days & 30 days & 17 months \\
\hline $\mathrm{S}_{2}$ & $1.5 \times 10^{18}$ & $2.4 \times 10^{17}$ & $1.2 \times 10^{17}$ & $8.8 \times 10^{16}$ & $6.7 \times 10^{16}$ & $8.9 \times 10^{15}$ \\
\hline $\mathrm{S}_{8}$ & - & $2.7 \times 10^{17}$ & $3.4 \times 10^{17}$ & $3.5 \times 10^{17}$ & $3.5 \times 10^{17}$ & $3.5 \times 10^{17}$ \\
\hline $\mathrm{H}_{2} \mathrm{~S}$ & $7.7 \times 10^{16}$ & $7.4 \times 10^{16}$ & $4.4 \times 10^{15}$ & $2.0 \times 10^{14}$ & $3.9 \times 10^{13}$ & $6.2 \times 10^{13}$ \\
\hline $\mathrm{H}_{2} \mathrm{~S}_{4}$ & - & $6.0 \times 10^{13}$ & $5.9 \times 10^{14}$ & $5.9 \times 10^{14}$ & $5.9 \times 10^{14}$ & $5.9 \times 10^{14}$ \\
\hline $\mathrm{CS}$ & $1.4 \times 10^{12}$ & $1.6 \times 10^{14}$ & $2.3 \times 10^{15}$ & $5.7 \times 10^{15}$ & $1.7 \times 10^{16}$ & $1.1 \times 10^{17}$ \\
\hline $\mathrm{CS}_{2}$ & $2.0 \times 10^{15}$ & $1.9 \times 10^{15}$ & $4.7 \times 10^{14}$ & $1.3 \times 10^{14}$ & $4.1 \times 10^{13}$ & $1.6 \times 10^{14}$ \\
\hline $\mathrm{H}_{2} \mathrm{CS}$ & - & $1.0 \times 10^{14}$ & $1.2 \times 10^{15}$ & $9.9 \times 10^{14}$ & $6.8 \times 10^{14}$ & $1.0 \times 10^{14}$ \\
\hline $\mathrm{CH}_{3} \mathrm{SH}$ & - & $2.1 \times 10^{11}$ & $6.6 \times 10^{12}$ & $9.9 \times 10^{12}$ & $1.5 \times 10^{13}$ & $9.0 \times 10^{11}$ \\
\hline $\mathrm{C}_{2} \mathrm{H}_{4} \mathrm{~S}$ & - & $2.2 \times 10^{12}$ & $3.8 \times 10^{12}$ & $3.3 \times 10^{12}$ & $2.1 \times 10^{12}$ & $1.2 \times 10^{11}$ \\
\hline NS & - & $2.3 \times 10^{14}$ & $3.0 \times 10^{16}$ & $5.9 \times 10^{16}$ & $6.8 \times 10^{16}$ & $6.1 \times 10^{16}$ \\
\hline HNCS & - & $4.5 \times 10^{13}$ & $7.7 \times 10^{15}$ & $2.0 \times 10^{16}$ & $2.7 \times 10^{16}$ & $3.2 \times 10^{16}$ \\
\hline
\end{tabular}

Times refer to approximate times after splashdown. $\mathrm{S}_{8}$ and $\mathrm{H}_{2} \mathrm{~S}_{4}$ will be primarily in the condensed phase. 
West et al. [1995] claim that the visual properties of the aerosols do not change appreciably with time, except for changes that are consistent with the particle size increasing due to coagulation. This result is somewhat surprising since one might naively expect the continual accumulation of $\mathrm{S}_{8}$ to brighten the aerosols over time - our predicted condensation flux for $\mathrm{S}_{8}$ (as much as $1 \times 10^{-12} \mathrm{~g} \mathrm{~cm}^{-2} \mathrm{~s}^{-1}$ ) is enormous. Perhaps small amounts of impurities have darkened the elemental sulfur. Alternatively, signatures for $S_{8}$ condensing about a darker particle might not show up clearly pure $S_{8}$ is very transparent at yellow-to-red and nearinfrared wavelengths. Detailed modeling of the optical properties of relevant composite mixtures and a further examination of all the available data might help resolve this issue. Note that $S_{8}$ absorbs strongly in the ultraviolet; future photochemicals models should consider the effects of UV-shielding by the aerosols.

Because we have assumed that the impact debris was mixed down to a few mbar at most and because the low eddy diffusion coefficients we adopt do not allow for appreciable mixing in the lower stratosphere, the only molecules that can condense in our model are species of low volatility and reasonable abundance. The only sulfur-containing molecules that fit this description (other than $\mathrm{S}_{8}$ ) are $\mathrm{H}_{2} \mathrm{~S}_{4}$ and higher-order sulfanes. We were unable to discover a molecule that forms dark aerosols yet that is volatile enough to account for the transient atmospheric ring features that several researchers suggest might be due to condensation and evaporation during the passage of an atmospheric wave.

\section{Summary and Conclusions}

In our model, sulfur photochemistry at the SL9 impact sites is rapid and complex. Condensed $S_{8}$ is the major reservoir for sulfur after the first few hours, but CS and nitrogen-sulfur species may also become important after several days, and the inventories of sulfanes $\left(\mathrm{H}_{2} \mathrm{~S}_{\mathrm{x}}\right)$ and thioformaldehyde $\left(\mathrm{H}_{2} \mathrm{CS}\right)$ are nontrivial. The observed condensates should be rich in sulfur. The photochemical model predictions from Table 1 can be used in conjunction with observations to shed light on important photochemical processes in the post-impact. Jovian stratosphere. For instance, we predict that $S_{2}$ will persist in the stratosphere longer than its photolysis lifetime warrants. HST FOS spectra [Noll et al., $1995]$ indicate the presence of $S_{2}$ three days after impact but not 23 days later. Is our narrowly-confined $S_{2}$ profile consistent with these observations? If not, can that result tell us something about the rates of some unmeasured sulfur reactions? The CS variations observed at millimeter wavelengths [Lellouch et al., 1995] are affected by horizontal spreading as well as absolute abundances. Our results might help in the analysis of this complex behavior. Our prediction that $\mathrm{CS}_{2}$ is lost more rapidly than $\mathrm{NH}_{3}$ in the first month after the impacts [see Moses et al., 1995] is consistent with HST FOS observations [Yelle and McGrath, 1995]; however, we may be losing $\mathrm{CS}_{2}$ more rapidly in our model than it is lost on Jupiter. All of our conclusions are highly speculative and depend on several kinetic reaction rates and initial conditions that are unknown at this time. We will examine the sensitivity of our model to different parameters in a later work.

Acknowledgments. We are grateful to K. Zahnle and B. Fegley for useful discussions. This work was supported by the Lunar and Planetary Institute and represents LPI contribution number 858 . Support from NASA grant NAGW 1509 and NSF grant ATM-9322506 is also gratefully acknowledged.

\section{References}

Bjoraker, G. L., 'T. Herter, G. Gull, S. Stolovy, and B. Pirger, Detection of water in the fireball of fragments $\mathrm{G}$ and $\mathrm{K}$ of Comet Shoemaker-Levy 9, Bull. Am. Astron. Soc., 26, 1578-1579, 1994.

Gladstone, G. R., M. Allen, and Y. L. Yung, Hydrocarbon photochemistry in the upper atmosphere of Jupiter, manuscript in preparation, 1995.

Khare, B. N., C. Sagan, E. L. Bandurski, and B. Nagy, Ultraviolet photoproduced organic solids synthesized under simulated Jovian conditions: molecular analysis, Science, $199,1199-1201,1978$.

Lellouch, E., et al., Chemical and thermal response of Jupiter's atmosphere following the impact of comet Shoemaker-Levy 9, Nature, 373, 592-595, 1995.

Meadows, V., D. Crisp, G. Orton, T. Brooke, and J. Spencer, AAT observations of Shoemaker Levy-9 collisions with Jupiter, Bull. Am. Astron. Soc., 26, 1588, 1994.

Moses, J. I., M. Allen, and G. R. Gladstone, Nitrogen and oxygen photochemistry following SL9, Geophys. Res. Lett., 'This issue, 1995.

Noll, K. S., et al., Hubble Space Telescope spectroscopic observations of Jupiter after the impact of Comet SL9, Bull. Am. Astron. Soc., 26, 1576-1577, 1994.

Noll, K. S., et al., Hubble Space Telescope spectroscopic observations of Jupiter after the collision of Comet P/Shoemaker-Levy 9, Science, 267, 1307-1313, 1995.

Orton, $\mathrm{G}$, et al., Jovian atmospheric structure investigation in the NASA IRTF SL9 campaign, Bull. Am. Astron. Soc., 26, 1581, 1994 .

West, R. A., et al., Impact debris particles in Jupiter's stratosphere, Science, 267, 1296-1301, 1995.

Yelle, R. V., and M. A. McGrath, Ultraviolet spectroscopy of the SL9 impact sites, l: The $175-230 \mathrm{~nm}$ region, Submitted to Icarus, 1995.

Yung, Y. I., M. Allen, and J. P. Pinto, Photochemistry of the atmosphere of Titan: Comparison between model and observations, Astrophys. J.Suppl. Ser., 55, 465-506, 1984.

Zahnle, K., M.-M. MacLow, K. Lodders, and B. F. Fegley, Jr., Sulfur chemistry in the wake of Comet ShoemakerLevy 9, Geophys. Res. Lett., In press, 1995.

M. Allen, JPL 183-601, Pasadena, CA 91109.

G. R. Gladstone, Southwest Research Institute, PO

Drawer 28510, 6220 Culebra Rd., San Antonio, TX 78284

J. I. Moses, Lunar and Planetary Institute, 3600 Bay

Area Blvd., Houston, TX 77058

(Received December 29, 1994; revised March 10, 1995; accepted March 24, 1995.) 